\title{
Equal variations of Fermi level and work function in graphene at the nanoscale
}

\author{
Sayanti Samaddar, ${ }^{\dagger, \ddagger}$ Johann Coraux, ${ }^{\dagger, \ddagger}$ Sylvain C. Martin, ${ }^{\dagger, \ddagger}, \|$ Benjamin \\ Grévin, ${ }^{\dagger, \boldsymbol{\Phi}, \S}$ Hervé Courtois, ${ }^{\dagger, \ddagger}$ and Clemens B. Winkelmann* ${ }^{*}, \dagger$ \\ †Université Grenoble Alpes, F-38000 Grenoble, France \\ $\ddagger C N R S$, Institut Néel, F-38000 Grenoble, France \\ ฯCNRS Alpes, F-38000 Grenoble, France \\ $\S C E A, I N A C-S P r A M$, F-38000 Grenoble, France \\ \|CEA, INAC-SBT, F-38000 Grenoble, France \\ E-mail: clemens.winkelmann@neel.cnrs.fr
}

\begin{abstract}
If surface effects are neglected, any change of the Fermi level in a semiconductor is expected to result in an equal and opposite change of the work function. This is however not observed in general in three-dimensional semiconductors, because of Fermi level pinning at the surface. By combining Kelvin Probe Force Microscopy and Scanning Tunneling Spectroscopy on single layer graphene, we measure both the local work function and the charge carrier density. The one-to-one equivalence of changes in the Fermi level and the work function is demonstrated to accurately hold in single layer graphene down to the nanometer scale.
\end{abstract}




\section{Keywords}

graphene, work function, contact potential difference, scanning tunneling microscopy, Kelvin probe force microscopy, inhomogeneous doping

In the basic picture of semiconductors and semiconductor interfaces, the Fermi level position and the work function are utterly linked. ${ }^{1-5}$ The work function $W$ is defined as the difference of local vacuum energy level $E_{\mathrm{vac}}$ and the Fermi level $E_{\mathrm{F}}$. If $E_{\mathrm{vac}}$ is fixed, one thus has simply $\partial W / \partial E_{\mathrm{F}}=-1$. This fundamental relation however fails to hold at the surface of most three-dimensional semiconductors owing to the pinning of the Fermi level by surface states. As sketched in Fig. 1, a generally large density of surface states lying within the semiconductor band gap pins the Fermi level with respect to the band structure. Any change in $E_{F}$ is thereby accompanied by an almost equal shift in the band structure and thus $E_{\mathrm{vac}}$ at the surface, leading to $\left|\partial W / \partial E_{\mathrm{F}}\right| \ll 1$ in practice. $^{3}$

Graphene was the first semi-metal for which the charge carrier density (or electronic doping) could be widely tuned. ${ }^{6,7}$ Along with other organic semiconductors ${ }^{8}$ or lamellar materials, such as transition metal dichalcogenides, the graphene surface has no dangling bonds that provide surface states to pin the Fermi level. Eventually it was shown that electronic doping has a significant effect on the work-function in single layer graphene, ${ }^{9-11}$ an effect which becomes weaker for multilayer graphene samples. ${ }^{12-14}$ By electrostatically tuning the doping level in a graphene device, the work function was observed to follow with good agreement the expected $\partial W / \partial E_{\mathrm{F}}=-1$ gate dependence, with $E_{D}-E_{F}\left(E_{D}\right.$ being the Dirac point energy in the graphene band structure) assessed from transport measurements through the device. ${ }^{9,11}$ The above conclusions were thus drawn from values of both $W$ and $E_{D}-E_{F}$ obtained from spatial averaging over the entire device, without demonstrating the work function to doping level correspondence on the local scale. However, several recent studies have unveiled that both the surface potential and the doping level in graphene are subject to strong variations on the nanometre scale. ${ }^{14-20}$

Yet, the detailed understanding of the interplay of doping and work function is key for a 


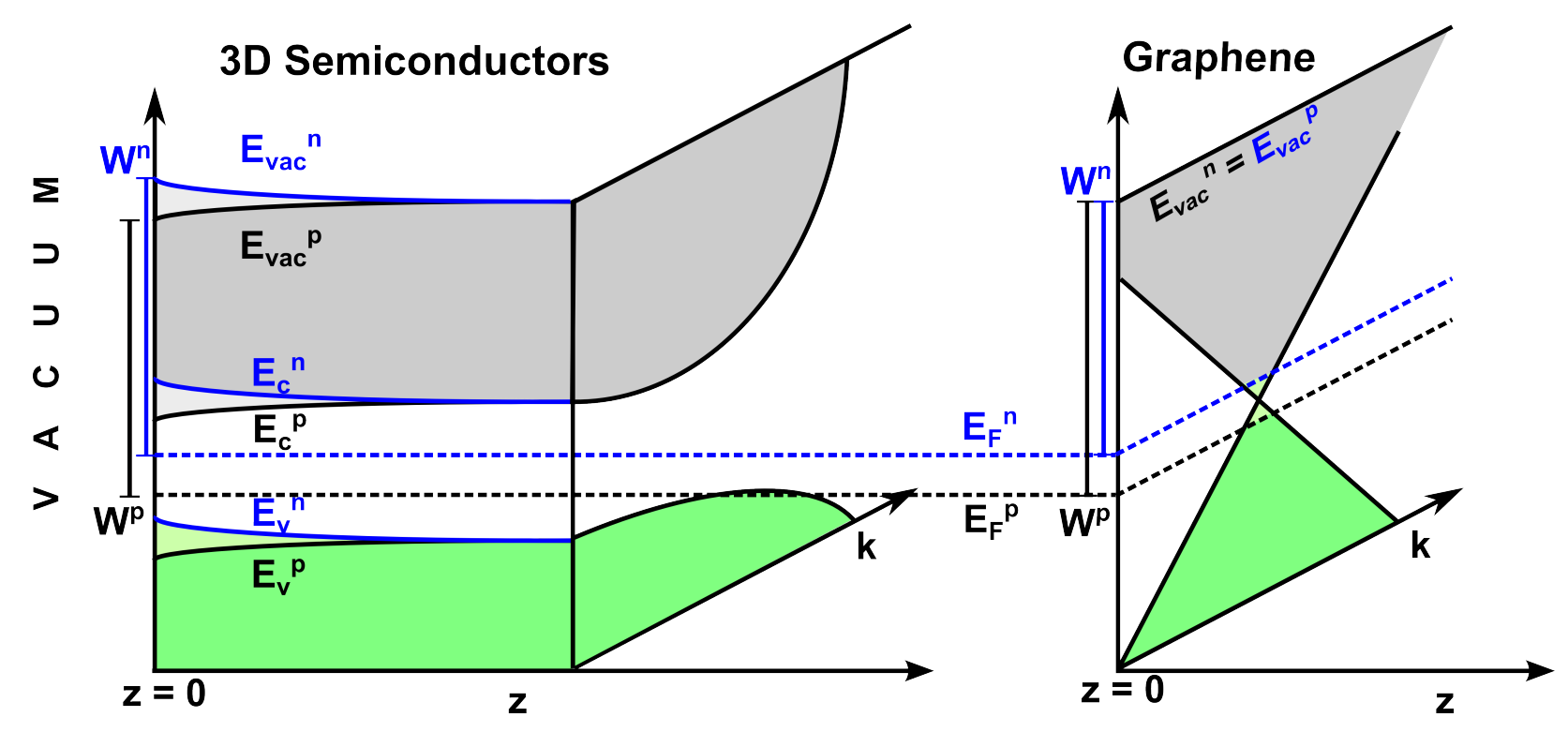

Figure 1: Sketch of the band structure and its filling in a three-dimensional semiconductor (left) and in graphene (right), as a function of wave vector and of distance normal to the vacuum interface. The surface states of the $3 \mathrm{D}$ semiconductor are not represented. $E_{v}, E_{c}$ and $E_{\mathrm{vac}}$ are the local values of the top of the valence band, the bottom of the conduction band, and the vacuum energy, respectively. The superscripts $n$ and $p$ indicate the situation of electron and hole doping, respectively.

large range of applications in electron field emission devices and optoelectronics. Downscaling the graphene work function control to the nanometer range can be highly advantageous, for example in the prospect of high-resolution and efficient field emission array-based devices, as was strikingly illustrated for carbon nanotubes. ${ }^{21-23}$ Because of charge disorder, the doping level in graphene itself can be strongly inhomogeneous down to the nanometer length scale, ${ }^{15-17,19,20,24}$ calling thus for a truly local comparison of the Dirac point level (that is, doping) and work function.

\section{Results and discussion}

Here, we use scanning probe microscopy - Scanning tunneling microscopy (STM) and Kelvin probe force microscopy (KPFM) - to experimentally compare the local electronic charge carrier density (that is, the Fermi level position) and the work function in single layer graphene. 

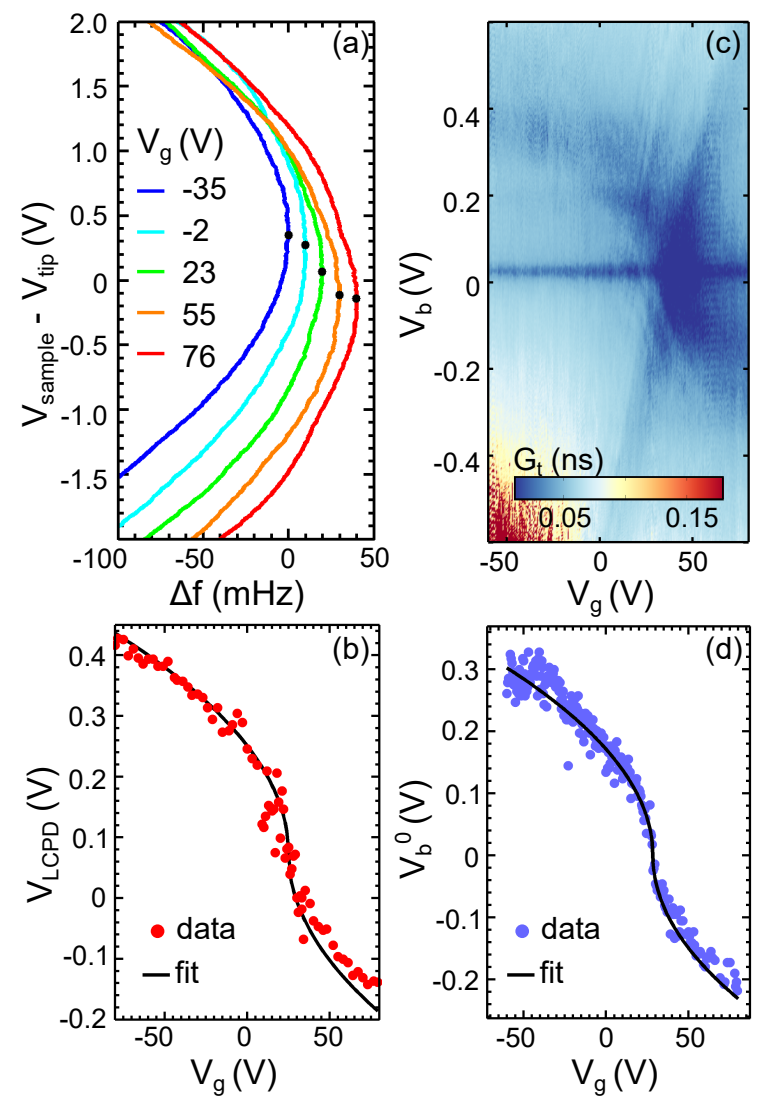

Figure 2: (a) Variation of the AFM probe frequency shift (each curve is offset by $10 \mathrm{mHz}$ for clarity) with sample-to-tip voltage, at different back-gate voltages $V_{g}$. The black dots indicate the position of the curves' maxima at $V_{\mathrm{LCPD}}$, as determined from a parabolic fit. (b) Change of $V_{\mathrm{LCPD}}$ with $V_{g}$. The line shows the best fit (see text) with fit parameters $V_{g}^{D}=25 \mathrm{~V}$ and $W_{0}=80 \mathrm{meV}$. (c) Color map showing the dependence of the differential tunneling conductance $G_{t}$ on bias and back gate voltage. (d) Back-gate voltage dependence of the primary minimum $V_{b}^{0}$ (see text) of the tunnel spectroscopies as shown in (c). The line shows the fit with Eq. (1), yielding $V_{g}^{D}=28 \mathrm{~V}$.

Hereto, we study the conjunct variations of the above quantities as a function of (i) electrostatic gating and (ii) position across local delaminations of the graphene from its substrate. We have studied two different kinds of graphene samples. The first set of data presented here was obtained on graphene prepared by mechanical exfoliation on $\mathrm{SiO}_{2} / \mathrm{Si}^{6}{ }^{6}$ We further investigate graphene grown on an $\operatorname{Ir}(111)$ substrate and decoupled by intercalation of a disordered molecular layer. ${ }^{19,25}$ We demonstrate in both cases that variations of the local charge neutrality point, $E_{D}-E_{F}$, are in one-to-one correspondence with variations of the work function. 
In the presence of a back-gate potential $V_{g}$, the carrier density in graphene can be adjusted according to $n=\alpha\left(V_{g}-V_{g}^{D}\right)$, where $V_{g}^{D}$ is an offset gate voltage compensation for a nonneutral charge background. Employing a parallel plate-capacitor model, the prefactor writes $\alpha=\kappa \epsilon_{0} /$ et, with $-e$ the charge of the electron, $\epsilon_{0}=8.85 \times 10^{-12} \mathrm{Fm}^{-1}$ the free space permittivity, $t=285 \mathrm{~nm}$ and $\kappa=3.9$ the thickness and dielectric constant of $\mathrm{SiO}_{2}$ in the substrate respectively. The distance of the graphene Dirac point to the Fermi level, $E_{\mathrm{D}}-E_{\mathrm{F}}$, is then given by

$$
E_{\mathrm{D}}-E_{\mathrm{F}}=-\hbar v_{\mathrm{F}} \operatorname{sign}\left(V_{g}-V_{g}^{D}\right) \sqrt{\pi \alpha\left|V_{g}-V_{g}^{D}\right|}
$$

with $v_{F}=1.1 \times 10^{6} \mathrm{~m} / \mathrm{s}$ the graphene Fermi velocity on $\mathrm{SiO}_{2} \cdot{ }^{26}$

Scanning tunneling microscopy and spectroscopy can provide a sensitive measurement of the local doping level in graphene. ${ }^{16}$ Indeed, at low temperature, the differential tip-tosample tunneling conductance $G_{t}=\partial I_{t} / \partial V_{b}$ is proportional to the local density of states of the sample at energy $e V_{b} \cdot{ }^{27}$ To first approximation, in tunneling spectroscopy on graphene, a V-shaped curve is hence expected, whose minimum occurs at a bias $V_{b}^{0}=\left(E_{\mathrm{D}}-E_{\mathrm{F}}\right) / e$. Measurements of the spatial variations of $V_{b}^{0}$ have been widely used for the study of doping disorder in graphene. ${ }^{16,17,19,20}$ Refinements of the above description take into account an additional and local field effect produced by the tip, ${ }^{28,29}$ which can eventually lead to electronic confinement below the tip apex. ${ }^{30}$ Because the tip is grounded, the larger the tip-sample capacitance, the larger the value of the back-gate voltage (in absolute values) required for locally cancelling the non-neutral charge background.

KPFM is performed at slightly larger tip-sample distances, such that tunneling can be neglected and van der Waals forces are small. In frequency-modulated non-contact atomic force microscopy and related techniques such as KPFM, measuring the resonator frequency shift $\Delta f$ provides access to the mechanical tip-sample force gradients. ${ }^{31}$ At zero bias voltage, that is, when the Fermi levels of probe and sample are aligned, an electric field exists between probe and sample, due to their distinct work functions and, in some cases, to local charges or dipoles. The local contact potential difference $\left(V_{\mathrm{LCPD}}\right)$ is the potential of the sample 
with respect to the tip that allows for cancelling these electrostatic forces. In the absence of isolated charges or dipoles, this potential equals the difference in the sample and tip work functions, ${ }^{32} V_{\mathrm{LCPD}}=\left(W_{\text {sample }}-W_{\text {tip }}\right) / e$.

Using KPFM in situ on the same sample and graphene area, we have recorded the parabolic dependence of $\Delta f$ on $V_{b}$ for different gate voltages, the maximum of each parabola corresponding to $V_{b}=V_{\mathrm{LCPD}}$, whose dependence on $V_{g}$ is shown in Figs. 2a,b. It is nicely fitted by the right-hand term of Eq. (1) offset by the sample-tip work function difference at the graphene charge neutrality point, $W_{0}$. The small value of $\left|W_{0}\right|<0.1 \mathrm{eV}$ is in good agreement with the fact that both the tungsten tip and charge neutral graphene have a work function close to $4.5 \mathrm{eV} .{ }^{33}$

Tunnel spectroscopies acquired in situ in the immediate vicinity (Figs. 2c,d) show an identical gate dependence of $e V_{b}^{0}$, that is, $E_{D}-E_{F}$, which is also very well fitted by Eq. (1). Notably, the gate coupling $\alpha$ is the same for both experiments and is entirely determined from known parameters. The close agreement of both sets of data thus confirms the precise equivalence of changes of the Fermi level position and the work function in graphene. As compared to previous work, ${ }^{9}$ this is to our knowledge the first comparison of both the work function and the Fermi level determined on the local scale. In our experiment, the only difference between both approaches appears in the value of $V_{g}^{D}$. This can be well understood by considering the fact that the tip-sample capacitance being an order of magnitude larger in STM contact, the tip acts as a strong local gate which locally distorts the doping profile. Apart from the tip-sample capacitance, the extent of this distortion directly depends on the effective potential drop between the sample and tip which is equal to $V_{b}-V_{\mathrm{LCPD}}$. This would ultimately cause a slight shift in the point of occurrence of charge neutrality, which depends on the tip-sample distance and the work function mismatch between charge neutral graphene and the tip. ${ }^{20}$

We now turn to the discussion of the relative variations of work function and $E_{D}-E_{F}$ in a different configuration, namely, graphene decoupled from the iridium substrate it was 

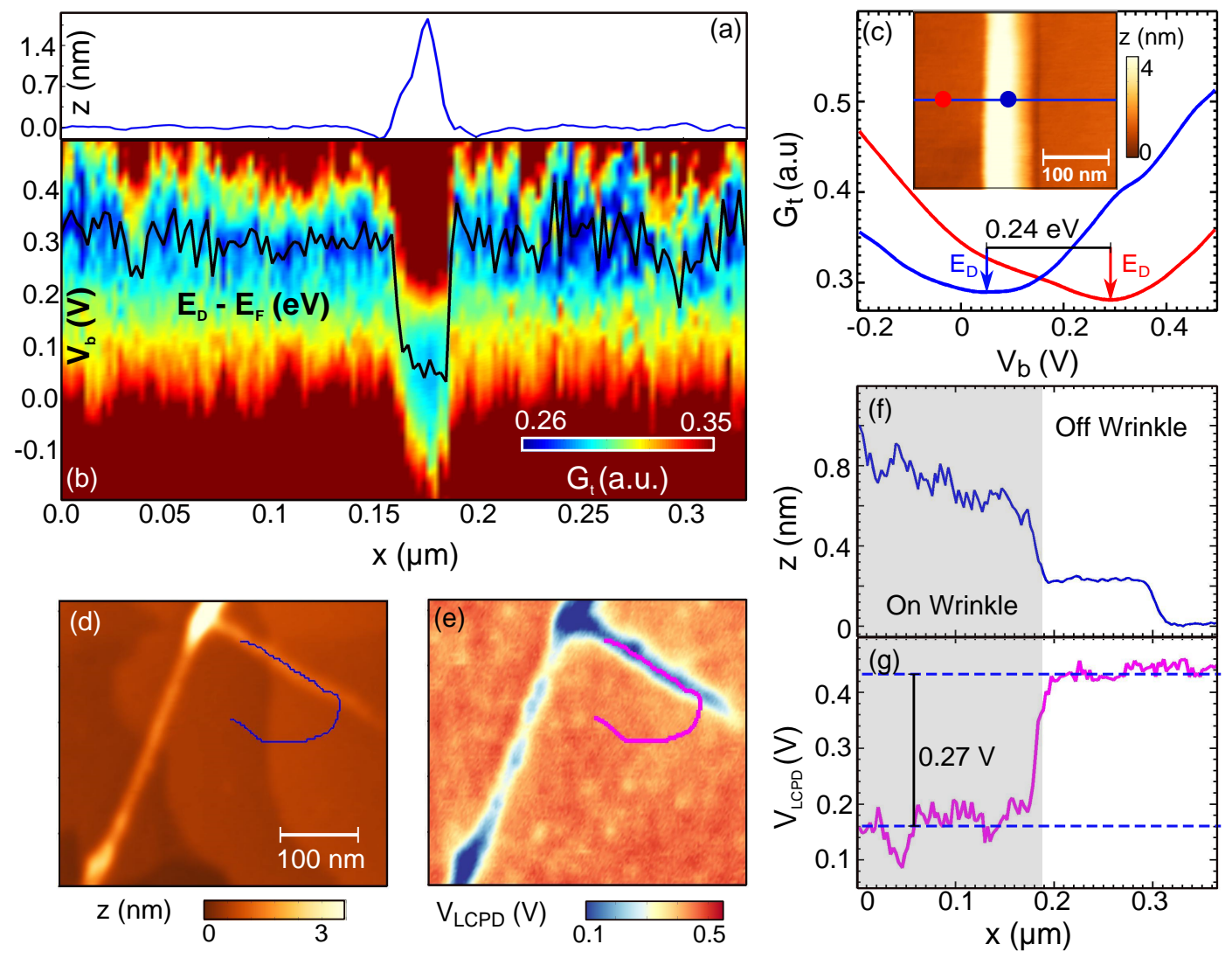

Figure 3: (a) STM height profile and (b) color map of the tunneling conductance along a line crossing a wrinkle. The black line follows the minima of the spectra, yielding the local value of $E_{D}-E_{F}$. (c) Typical differential tunneling conductance $G_{t}$ spectra on (blue) and off (red) the same graphene wrinkle, taken at the positions marked by points of corresponding colors in the inset (STM image of the wrinkle). (d) AFM topograph and (e) simultaneously acquired $V_{\mathrm{LCPD}}$ map of decoupled graphene on $\operatorname{Ir}(111)$. UHV, room temperature, imaging parameters: $\Delta f=-15 \mathrm{~Hz}$, cantilever oscillation amplitude $A=8.4 \mathrm{~nm}$. (f) Height profile of a graphene wrinkle along the blue path shown in (d). (g) $V_{\text {LCPD }}$ profile over the same path, marked by the magenta line in (e).

grown on. This electronic decoupling occurs as a consequence of intercalation of oxygen-rich species in ambient air conditions. ${ }^{25}$ By scanning tunneling spectroscopy on flat graphene, we find a Fermi level about $300 \mathrm{meV}$ below the Dirac point (Fig. 3g), in agreement with previous reports, ${ }^{19}$ which corresponds to a hole density of about $9 \times 10^{11} \mathrm{~cm}^{-2}$. Here, one cannot adjust the average charge carrier density by electric field effect from a back gate. However, one can correlate spatial variations of the Dirac point level and the work function along the sample surface by moving to topographic delaminations of the graphene, the so- 
called wrinkles. ${ }^{34}$ Notably, when scanning across a graphene delamination, the tunneling spectroscopies are found to be qualitatively unchanged, except for a marked variation of the bias voltage $V_{b}^{0}$ at the spectra's minima (Fig. 3a-c). From a statistical analysis over several wrinkles, we find that the otherwise strongly $p$-doped graphene is close to charge neutral when delaminated, with a hole density $<10^{10} \mathrm{~cm}^{-2}$. As shown for a typical situation in Fig. 3c, we find an average reduction of $E_{D}-E_{F}$ by $240 \pm 50 \mathrm{meV}$ when moving to the wrinkle. This reduced $p$ doping can be explained by the larger distance (by about $2 \mathrm{~nm}$ ) of the graphene sheet to the charges at the substrate.

Using KPFM we have mapped the work function on a nominally identical sample. In Fig. 3d-g we follow the $V_{\mathrm{LPCD}}$ as the tip moved from flat graphene to a wrinkle. An abrupt decrease of $V_{\mathrm{LPCD}}$ by $270 \pm 60 \mathrm{mV}$ is observed. Similar results were obtained on series of other wrinkles. Here again, the combination of both techniques leads to conclude on the precise correspondence in graphene between variations of the Fermi level position and the work function, within an experimental precision of about $\pm 10 \%$. Notably, both quantities are observed to vary accordingly with a spatial resolution below $10 \mathrm{~nm}$.

\section{Conclusion}

In conclusion, by combining two scanning probe microscopies, we have demonstrated the one-to-one correspondence of the Fermi level and the work function in graphene to hold accurately and on the local scale. We also stress that our methodology is of general relevance for the ever-growing family of two-dimensional materials, for which a precise knowledge of the local electronic properties is crucial if next generation optoelectronic devices based on them are to be designed. Further, small topographic features in graphene such as delaminations are prototypical examples of ultimately efficient field emitters. We propose that arrays of graphene wrinkles be considered in ultra-high density field emitters with adjustable efficiency. 


\section{Methods}

To allow for charge carrier evacuation, the graphene flakes in the graphene/ $\mathrm{SiO}_{2}$ samples were contacted by lateral electrodes using a stencil technique. ${ }^{20}$

The graphene/ $\operatorname{Ir}(111)$ samples were grown by thermally-induced catalytic cracking of ethylene molecules at the hot surface of $\operatorname{Ir}(111)$ under ultra-high vacuum (UHV) conditions. ${ }^{35}$ Iridium thin films terminated by a (111) surface, of $10 \mathrm{~nm}$ thickness, were prepared on a sapphire substrate ${ }^{36}$ and used a support for the growth of graphene. Intercalation occurs gradually upon exposure to ambient conditions ${ }^{25}$ and leads to electronic decoupling of the graphene from its metallic substrate along with the appearance of sizable charge disorder. ${ }^{19}$

KPFM data on $\mathrm{Gr} / \mathrm{Ir}$ were obtained at room temperature in the regime of large oscillation amplitudes by using a VT-beam AFM system from Omicron (base pressure below $10^{-10}$ mbar) and silicon cantilevers (Nanoworld, typical spring constant k of $42 \mathrm{~N} . \mathrm{m}^{-1}$, resonance frequency around $300 \mathrm{kHz}$ ). The probes were in situ treated by thermal annealing and Ar sputtering. Topographic imaging was performed in the frequency modulation mode (FMAFM), and KPFM data were simultaneously recorded in the frequency modulation mode (FM-KPFM) with bias modulations of $1 \mathrm{~V}$ peak-to-peak.

STM and combined STM-KPFM were obtained with a cryogenic combined AFM-STM, ${ }^{19}$ based on a quartz length extension mechanical resonator. ${ }^{20,37-39}$ Such resonators can be allelectrically operated, are space-conserving and display a large effective stiffness $(1 \mathrm{MN} / \mathrm{m})$ along with quality factors $>10^{5}$ at low temperatures. The metallic tips were made out of $\mathrm{W}$ wires and sharpened by focussed ion beam. Scanning tunneling spectroscopy (STS) was performed via lock-in measurements of the differential tip-to-sample tunneling conductance $G_{t}=\partial I_{t} / \partial V_{b}$, by adding a $6 \mathrm{mV}$ ac modulation at $406 \mathrm{~Hz}$ to the static sample bias voltage $V_{b}$. For KPFM measurement on graphene on $\mathrm{SiO}_{2}$, the tip was retracted $5 \mathrm{~nm}$ above the surface as to minimize van der Waals contributions to the frequency shift. 


\section{Acknowledgement}

This work was funded by the European Commission under project no. 264034 (Q-NET Marie Curie Initial Training Network). Samples were fabricated at the Nanofab facility at Institut Néel. We thank L. Nony, P. Muret, B. Sacépé, A. De Cecco, and J. Seidemann for discussions and help with the experiments.

\section{References}

1. Bardeen, J. Surface States and Rectification at a Metal Semi-Conductor Contact. Phys. Rev. 1947, 71, 717-727.

2. Tersoff, J. Schottky barrier heights and the continuum of gap states. Phys. Rev. Lett. 1984, 52, 465-468.

3. Mönch, W. Semiconductor Surfaces and Interfaces; Springer, 1993.

4. Cahen, D.; Kahn, A. Electron energetics at surfaces and interfaces: concepts and experiments. Adv. Mater. 2003, 15, 271-277.

5. Kahn, A. Fermi level, work function and vacuum level. Mater. Horiz. 2016, 3, 7-10.

6. Novoselov, K. S.; Geim, A. K.; Morozov, S. V.; Jiang, D.; Zhang, Y.; Dubonos, S. V.; Grigorieva, I. V.; Firsov, A. A. Electric Field Effect in Atomically Thin Carbon Films. Science 2004, 306, 666-669.

7. Ohno, Y.; Maehashi, K.; Yamashiro, Y.; Matsumoto, K. Electrolyte-gated graphene field-effect transistors for detecting $\mathrm{pH}$ and protein adsorption. Nano Lett. 2009, 9, $3318-3322$.

8. Hill, I. G.; Milliron, D.; Schwartz, J.; Kahn, A. Organic semiconductor interfaces: electronic structure and transport properties. Appl. Surf. Science 2000, 166, 354-362. 
9. Yu, Y.-J.; Zhao, Y.; Ryu, S.; Brus, L. E.; Kim, K. S.; Kim, P. Tuning the graphene work function by electric field effect. Nano Lett. 2009, 9, 3430-3434.

10. Shi, Y.; Kim, K. K.; Reina, A.; Hofmann, M.; Li, L.-J.; Kong, J. Work Function Engineering of Graphene Electrode via Chemical Doping. ACS Nano 2010, 4, 2689-2694.

11. Yuan, H.; Chang, S.; Bargatin, I.; Wang, N. C.; Riley, D. C.; Wang, H.; Schwede, J. W.; Provine, J.; Pop, E.; Shen, Z.-X. et al. Engineering Ultra-Low Work Function of Graphene. Nano Lett. 2015, 15, 6475-6480.

12. Datta, S. S.; Strachan, D. R.; Mele, E. J.; Johnson, A. T. C. Surface Potentials and Layer Charge Distributions in Few-Layer Graphene Films. Nano Lett. 2009, 9, 7-11.

13. Lee, N. J.; Yoo, J. W.; Choi, Y. J.; Kang, C. J.; Jeon, D. Y.; Kim, D. C.; Seo, S.; Chung, H. J. The interlayer screening effect of graphene sheets investigated by Kelvin probe force microscopy. Appl. Phys. Lett. 2009, 95, 222107.

14. Ziegler, D.; Gava, P.; Güttinger, J.; Molitor, F.; Wirtz, L.; Lazzeri, M.; Saitta, A. M.; Stemmer, A.; Mauri, F.; Stampfer, C. Variations in the work function of doped singleand few-layer graphene assessed by Kelvin probe force microscopy and density functional theory. Phys. Rev. B 2011, 83, 235434.

15. Martin, J.; Akerman, N.; Ulbricht, G.; Lohmann, T.; Smet, J. H.; von Klitzing, K.; Yacoby, A. Observation of electron-hole puddles in graphene using a scanning singleelectron transistor. Nature Phys. 2008, 4, 144-148.

16. Zhang, Y.; Brar, V. W.; Girit, C.; Zettl, A.; Crommie, M. F. Origin of spatial charge inhomogeneity in graphene. Nature Phys. 2009, 5, 722-726.

17. Deshpande, A.; Bao, W.; Zhao, Z.; Lau, C. N.; LeRoy, B. J. Imaging charge density fluctuations in graphene using Coulomb blockade spectroscopy. Phys. Rev. B 2011, 83, 155409. 
18. Dedkov, Y.; Voloshina, E. Multichannel scanning probe microscopy and spectroscopy of graphene moiré structures. Phys. Chem. Chem. Phys. 2014, 16, 3894-3908.

19. Martin, S. C.; Samaddar, S.; Sacépé, B.; Kimouche, A.; Coraux, J.; Fuchs, F.; Grévin, B.; Courtois, H.; Winkelmann, C. B. Disorder and screening in decoupled graphene on a metallic substrate. Phys. Rev. B 2015, 91, 041406(R).

20. Samaddar, S.; Yudhistira, I.; Adam, S.; Courtois, H.; Winkelmann, C. B. Charge Puddles in Graphene near the Dirac Point. Phys. Rev. Lett. 2016, 116, 126804.

21. De Heer, W. A.; Chatelain, A.; Ugarte, D. A carbon nanotube field-emission electron source. Science 1995, 270, 1179-1180.

22. Collins, P. G.; Zettl, A. A simple and robust electron beam source from carbon nanotubes. Appl. Phys. Lett. 1996, 69, 1969-1971.

23. Saito, Y.; Hamaguchi, K.; Hata, K.; Uchida, K.; Tasaka, Y.; Ikazaki, F.; Yumura, M.; Kasuya, A.; Nishina, Y. Conical beams from open nanotubes. Nature 1997, 389, 554555.

24. Das Sarma, S.; Adam, S.; Hwang, E. H.; Rossi, E. Electronic transport in twodimensional graphene. Rev. Mod. Phys. 2011, 83, 407-470.

25. Kimouche, A.; Renault, O.; Samaddar, S.; Winkelmann, C.; Courtois, H.; Fruchart, O.; Coraux, J. Modulating charge density and inelastic optical response in graphene by atmospheric pressure localized intercalation through wrinkles. Carbon 2014, 68, 73-79.

26. Jung, S.; Rutter, G. M.; Klimov, N. N.; Newell, D. B.; Calizo, I.; Hight-Walker, A. R.; Zhitenev, N. B.; Stroscio, J. A. Evolution of microscopic localization in graphene in a magnetic field from scattering resonances to quantum dots. Nature Phys. 2011, 7, $245-251$. 
27. Tersoff, J.; Hamann, D. Theory and application for the scanning tunneling microscope. Phys. Rev. Lett. 1983, 50, 1998-2001.

28. McEllistrem, M.; Haase, G.; Chen, D.; Hamers, R. J. Electrostatic sample-tip interactions in the scanning tunneling microscope. Phys. Rev. Lett. 1993, 70, 2471-2474.

29. Choudhary, S. K.; Gupta, A. K. Effects of tip induced carrier density in local tunnel spectra of graphene. Appl. Phys. Lett. 2011, 98, 102109.

30. Zhao, Y.; Wyrick, J.; Natterer, F. D.; Rodriguez-Nieva, J. F.; Lewandowski, C.; Watanabe, K.; Taniguchi, T.; Levitov, L. S.; Zhitenev, N. B.; Stroscio, J. A. Creating and probing electron whispering-gallery modes in graphene. Science 2015, 348, 672-675.

31. Sadewasser, S. In Kelvin Probe Force Microscopy; Sadewasser, S., Glatzel, T., Eds.; Springer Berlin Heidelberg, 2012; p 11.

32. Melitz, W.; Shen, J.; Kummel, A. C.; Lee, S. Kelvin probe force microscopy and its application. Surf. Sci. Rep. 2011, 66, 1-27.

33. Liang, S.-J.; Ang, L. K. Electron Thermionic Emission from Graphene and a Thermionic Energy Converter. Phys. Rev. Applied 2015, 3, 014002.

34. N'Diaye, A. T.; van Gastel, R.; Martinez-Galera, A. J.; Coraux, J.; Hattab, H.; Wall, D.; zu Heringdorf, F.-J. M.; von Hoegen, M. H.; Gómez-Rodríguez, J. M.; Poelsema, B. et al. In situ observation of stress relaxation in epitaxial graphene. New Jour. of Phys. 2009, $11,113056$.

35. Coraux, J.; N Diaye, A. T.; Busse, C.; Michely, T. Structural Coherency of Graphene on $\operatorname{Ir}(111)$. Nano Lett. 2008, 8, 565-570.

36. Vo-Van, C.; Kimouche, A.; Reserbat-Plantey, A.; Fruchart, O.; Bayle-Guillemaud, P.; Bendiab, N.; Coraux, J. Epitaxial graphene prepared by chemical vapor deposition on single crystal thin iridium films on sapphire. Appl. Phys. Lett. 2011, 98, 181903. 
37. Heike, S.; Hashizume, T. Atomic resolution noncontact atomic force/scanning tunneling microscopy using a 1 MHz quartz resonator. Appl. Phys. Lett. 2003, 83, 3620-3622.

38. An, T.; Nishio, T.; Eguchi, T.; Ono, M.; Nomura, A.; Akiyama, K.; Hasegawa, Y. Atomically resolved imaging by low-temperature frequency-modulation atomic force microscopy using a quartz length-extension resonator. Rev. of Sci. Instr. 2008, 79, 033703.

39. Giessibl, F. J.; Pielmeier, F.; Eguchi, T.; An, T.; Hasegawa, Y. Comparison of force sensors for atomic force microscopy based on quartz tuning forks and length-extensional resonators. Phys. Rev. B 2011, 84, 125409. 


\section{Graphical TOC Entry}

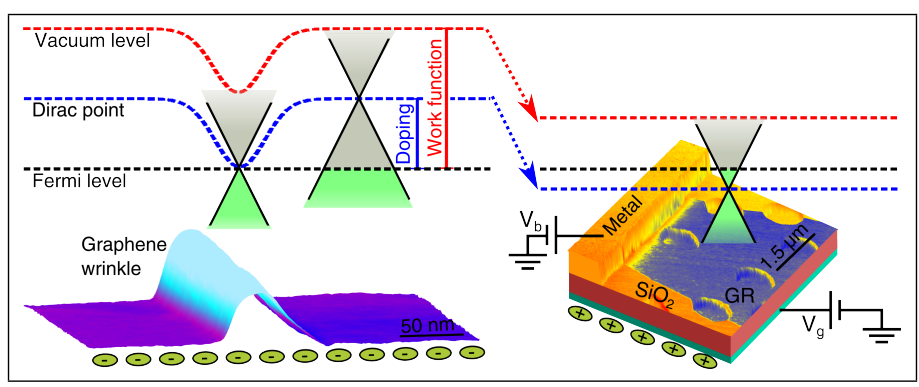

